\title{
Validation of myocardial damage biomarkers as reliable predictors of infarct size and ejection fraction impairment assessed by cardiac magnetic resonance
}

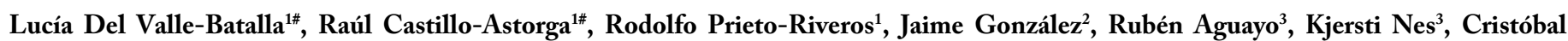
Ramos $^{4}$, Juan Carlos Prieto ${ }^{4}$ and Ramón Rodrigo ${ }^{2 *}$

${ }^{1}$ Medical students, Faculty of Medicine, University of Chile

${ }^{2}$ Molecular and Clinical Pharmacology Program, Institute of Biomedical Sciences, Faculty of Medicine, University of Chile

${ }^{3}$ San Juan de Dios Hospital, Santiago, Chile

${ }^{4}$ University of Chile Clinical Hospital, Santiago, Chile

\#Co-first authorship: These authors contributed equally to this work

\begin{abstract}
Background: Acute myocardial infarction (AMI) is the leading cause of morbidity and mortality worldwide. The final infarct size (FIS) and left ventricular ejection fraction (LVEF) are the greatest predictors of post-AMI mortality, with cardiac magnetic resonance (CMR) being the gold standard method for their measurement. Myocardial damage biomarkers, such as creatine kinase (CK) and myocardial creatine kinase (CKMB) are currently used to diagnose AMI and estimate the myocardial damage extent. It would be plausible to use them as predictors of FIS and LVEF; however, current evidence is not available up to date.

Objective: To determine the potential power of plasma CK and CKMB levels as predictors of FIS and LVEF impairment, respectively, on the basis of their correlation in patients undergoing primary coronary angioplasty (PCA) following ST-elevation acute myocardial infarction (STEMI).

Methodology: A retrospective analysis of PREVEC Trial (ISRCTN registry: 56034553), a multicentric, randomized, double-blind clinical study was performed. Sixty-seven patients with STEMI scheduled for PCA were enrolled. The CMR was performed 7-15 days after the event. Three radiologists blinded to clinical information measured FIS and LVEF. Total CK and CKMB were measured in peripheral venous blood at 6-8 hours after PCA. Correlation coefficient were obtained, and the tests were considered significant with a p value $<0.05$. The software GraphPrism 6.0 was used for the statistical analysis.
\end{abstract}

Results: A significant positive correlation was obtained between levels of cardiac biomarkers and FIS [total CK ( $r$-square 0.3 , p<0.0001) and CK MB ( $r$-square 0.15 , $\mathrm{p}<0.0027)$. In addition, the levels of these biomarkers showed a significant negative correlation with LVEF [total CK ( $r-s q u a r e ~ 0.3, p<0.0001)$ and CK MB ( $r-s q u a r e$ $0.18, \mathrm{p}<0.0012)]$

Conclusion: These results are consistent with the view that the myocardial damage biomarkers CK and CKMB are reliable as predictors of FIS and LVEF measured by CMR in post-AMI patients. These data suggest that these biomarkers could be included in future Risk Scores.

\section{Introduction}

Acute myocardial infarction (AMI) is the leading cause of morbidity and mortality in the world. It is estimated that 1 in 4 deaths worldwide are caused by it [1]. Following AMI, assessment of mortality risk is crucial for therapeutic decision making [2-4], currently performed through risk scores, such as TIMI, Grace, among others.

Myocardial damage biomarkers are used to diagnose AMI and estimate myocardial damage. Final infarct size (FIS) and left ventricular ejection fraction (LVEF) are variables that highly correlate with mortality, being cardiac magnetic resonance (CMR) the gold standard method for FIS and LVEF measurement [5].

The correlation of MDBs and infarct size is well established, contrary to the correlation with LVEF, which is the other huge predictor of mortality and cardiac function after AMI. Lower post-PCA peak of CKMB has been linked to a better improvement of LVEF after
13 months, proving to be one of the most strongly correlated predictors of LVEF in the study [6].

Also, peak concentrations of all three of the most used MDBs (CKMB, Troponins, and Total CK), had a very similar correlation with LVEF [7]. Retrospective analysis of clinical trials to assess correlation of infarct size and have been successfully used by Stanley Chia and colleagues, 2008 [8].

${ }^{\star}$ Correspondence to: Ramón Rodrigo, Medical students, Faculty of Medicine, University of Chile, Chile, E-mail: rrodrigo@med.uchile.cl

Key words: biomarkers of myocardial damage, cardiac magnetic resonance STEMI, left ventricular ejection fraction, final infarct size

Received: April 19, 2019; Accepted: April 26, 2019; Published: April 30, 2019 
Valle-Batalla LD (2019) Validation of myocardial damage biomarkers as reliable predictors of infarct size and ejection fraction impairment assessed by cardiac magnetic resonance

\section{Methodology}

\section{Study design and patient population}

A retrospective analysis of the clinical trial PREVEC (Prevention of reperfusion damage associated with percutaneous coronary angioplasty following acute myocardial infarction) was performed. PREVEC (ISRCTN registry: 56034553), a multicentric, randomized, doubleblind clinical trial, aimed to evaluate whether antioxidant vitamins $\mathrm{C}$ and $\mathrm{E}$ reduce infarct size in patients subjected to percutaneous coronary angioplasty after AMI [9]. Patients having experienced their first AMI, with ST-segment elevation (STEMI), and indication of primary percutaneous coronary angioplasty (PCA) were included after signing a written informed consent. The study was approved by the institutional review board and adhered to the declarations of Helsinki and Istanbul. The enrolment was performed between February 2013 and November 2015 in 3 cardiovascular units. The intervention in PREVEC consisted in the intravenous administration of high doses vitamin C; or placebo vehicle prior performing PCA, followed by oral administration of vitamin $C$ (Ascorbate, $500 \mathrm{mg} / 12 \mathrm{~h}$ ) and vitamin E ( $\alpha$-tocopherol, $400 \mathrm{IU} /$ day) for 84 days, or placebo. The exclusion criteria included a history of renal or hepatic insufficiency, heart failure (NYHA III or IV), cardiogenic shock, post-primary PCA TIMI grade flow $<3$, any serious medical comorbidity that indicated a life expectancy $<6$ months, pregnancy, history of renal stones, and glucose 6-phosphate dehydrogenase deficiency.

\section{Biomarkers}

Biomarkers were measured in blood samples drawn from the antecubital vein four times; before PCA (M1), immediately after reperfusion onset (M2), 6-8 hours after balloon deflation (M3), and
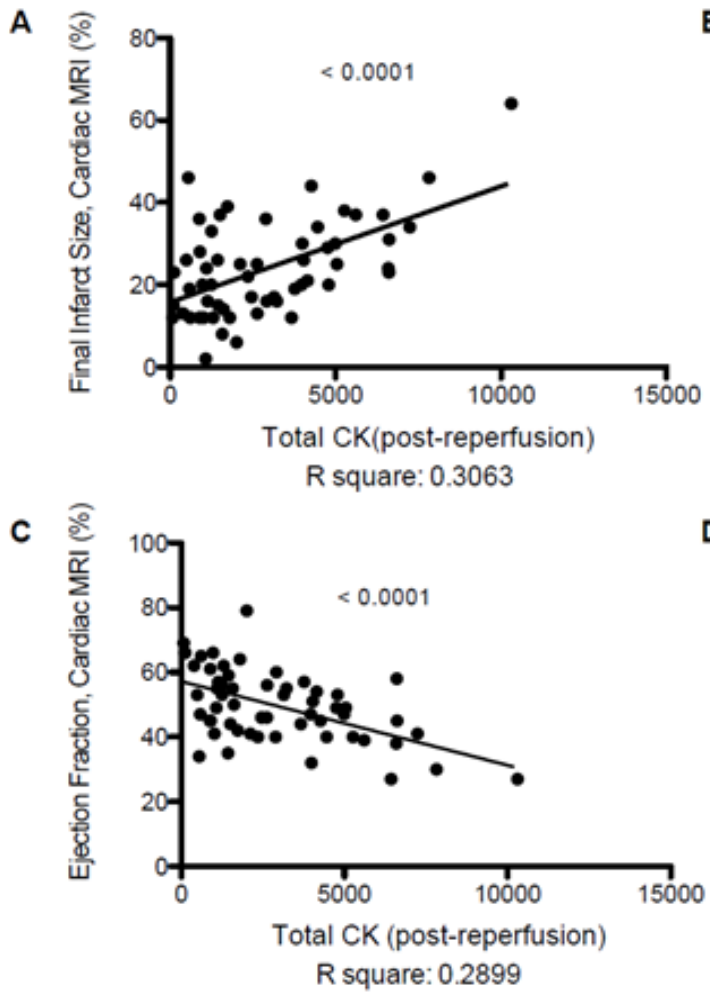

on hospital discharge (M4). The plasma total creatine kinase (CK) concentration and that of myocardial creatine kinase (CKMB) were measured using standard methods.

\section{Cardiac Magnetic Resonance}

Assessment through cardiac magnetic resonance (CMR) was performed at both 7-15 days and 2-3 months after PCA, measuring final infarct size (FIS), left ventricular ejection fraction (LVEF), and indexed end-systolic volume (IESV).

\section{Results}

There was no significant difference in infarct size between the groups either in the first or the second CMR (Fig. 1). In addition, LVEF and the IESV assessed by CMR also showed no significant difference between groups in the first or second CMR. The biomarkers blood samples were at 6-8 hours post successful reperfusion therapy to get close to the early peak concentration of MDBs.

A significant positive correlation was obtained between levels of cardiac biomarkers and FIS determined by CMR [total CK ( $r$-square $0.3, \mathrm{p}<0.0001)$ and CK MB ( $\mathrm{r}$-square $0.15, \mathrm{p}<0.0027)]$. In addition, the levels of these biomarkers showed a significant negative correlation with LVEF [total CK ( $r$-square 0.3, $\mathrm{p}<0.0001$ ) and CK MB (r-square $0.18, \mathrm{p}<0.0012)]$ (Figure 1).

\section{Discussion}

The present study contributes to validate the use of myocardial damage biomarkers as predictors of FIS and LVEF impairment in patients subjected to PCA following AMI.
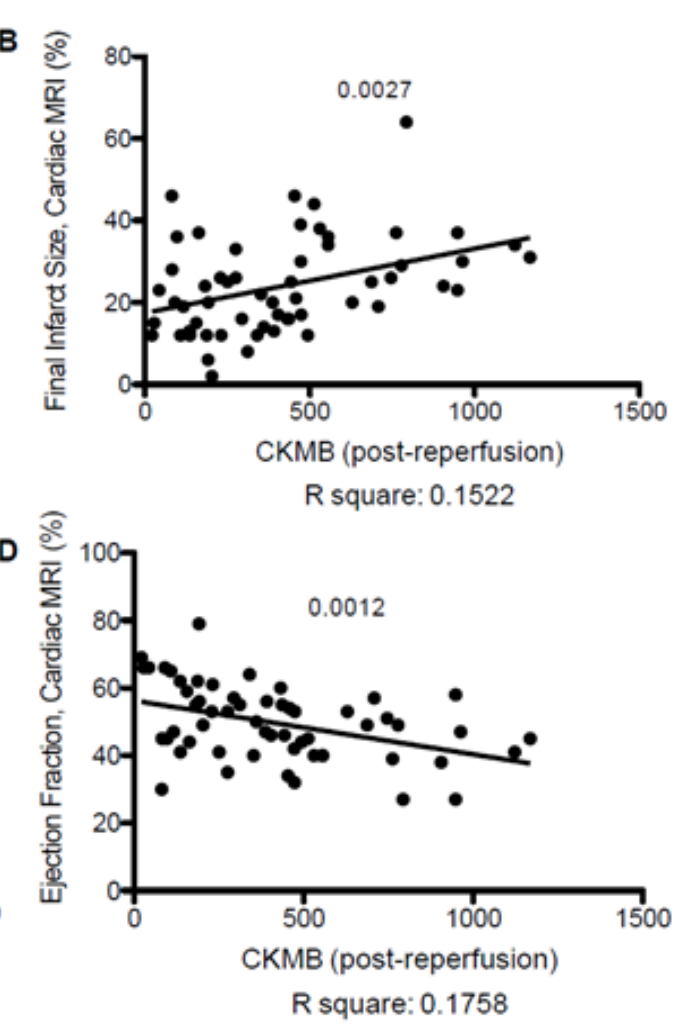

Figure1. A. Significant positive correlation between Total CK and FIS determined by CMR. (r-square $0.3, p<0.0001$ ).

B. Significant positive correlation between CKMB and FIS determined by CMR. ( $\mathrm{r}$-square $0.15, \mathrm{p}<0.0027$ ).

C. Significant negative correlation between Total CK and LVEF determined by CMR. ( $r$-square $0.3, p<0.0001$ ).

D. Significant negative correlation between CKMB and LVEF determined by CMR. ( $\mathrm{r}$-square $0.18, \mathrm{p}<0.0012$ ). 
Valle-Batalla LD (2019) Validation of myocardial damage biomarkers as reliable predictors of infarct size and ejection fraction impairment assessed by cardiac magnetic resonance

\section{MDBs and Imaging Parameters}

The main drawback of this study is the fact that only CKMB and total CK were measured. The addition of Troponins to the analysis would have been of great worth to compare the predictor potential of MDBs. Serial measurements of MDBs have been one of the most widely accepted methods for quantifying myocardial damage [10], hence, the significant positive correlation between Total CK/ CKMB and FIS determined by CMR was frankly expected. In turn, left ventricular function provides an assessment of the functional consequences of STEMI and ischemia. In this context, CMR has become the gold standard for determination of left ventricular volumes and LVEF [5,11]. This diagnostic modality appears to be better in terms of sensitivity, specificity, and reliability in detecting cardiac disorders associated with abnormal structure and function than other diagnostic techniques and does not involve exposure to ionizing radiation [12,13]. Despite the advantages of CMR in the clinical practice, the classical assessment of LVEF is currently made by echocardiography, according to recommendations on main guidelines [10] despite having several sources of error $[10,14]$.

LVEF assessment through MDBs was established in 1998, being measured by echocardiography [15] and since then several studies have correlated LVEF measured by Echo, and MDBs. The latest studies from 2017 had shown that biomarkers alone can be useful to determine the magnitude of left ventricular remodeling measured by CMR [7]. Although LVEF was not used as a direct outcome it is presumed that ventricular remodeling posterior to PCA involves changes with ejection fraction. Other studies suggest the same correlation on different age groups such as elderly patients [16]. Nevertheless, the true causal relationship is not sure to be established due to the fact that other items correlate the same way biomarkers do (leading to confusion bias). Finally, long-term studies have shown high sensitivity and specificity ( $90 \%$ and $73 \%$, respectively) at prediction of LVEF by MDBs, specifically high-sensitive cardiac troponin $\mathrm{T}$, compared to CMR up to one year after the event [17].

It is of interest to emphasize that apart from cardiac enzymes, several biomarkers have reached correlations with LVEF. Some of these are LDH and natriuretic peptides such as BNP and NT-proBNP $[17,18]$, thus supporting the feasibility for the determination of blood parameters in evaluating ventricular remodeling.

Due to the significant correlation between MDBs and LVEF measured by CMR, we propose that biomarkers would be of great value as a complement to the assessment of LVEF obtained by echocardiography. Thus, future studies are needed to know whether the added information of MDBs to echocardiography can assess LVEF better than echocardiography by its own. MDBs have been used complementary to echocardiography to assess chemotherapy damage in the treatment of cancer, as Echo alone is not sensitive enough to detect slight changes in LVEF [19].

\section{MDBs for Risk Assessment}

Although back in 1996, MDBs were established as powerful and independent risk predictors in AMI (Ohmna et al. 1996), now it is very controversial whether their prognostic value is enough to become value dependent variables in risk scores. MDBs recently proved not to be a predictor of major adverse cardiovascular and cerebrovascular events (MACCEs), and its prognostic value is being put into question [20].

In summary, the present data contribute to the view that myocardial damage biomarkers are reliable, easy to determine, and cost-effective tests, and are useful predictors of FIS and LVEF measured by CMR in post-AMI patients. Therefore, we think that the value of myocardial damage biomarkers could be of great worth in the clinical context. Furthermore, the addition of MDBs not only as dichotomic, but also as value dependent variables in risk scores (as it has been implemented with other variables in risk scores, such as Creatinine in GRACE Risk Score), could make future risk scores more accurate. There are many factors to consider to make this happen, and future research is needed to disentangle the real predictor potential of MDBs.

\section{Funding}

Supported by FONDECYT, grant 1120594.

\section{References}

1. https://www.cdc.gov/heartdisease/facts.htm

2. Antman EM, Cohen M, Bernink PJ, McCabe CH, Horacek T, et al. (2000) The TIMI risk score for unstable angina/non-ST elevation MI: A method for prognostication and therapeutic decision making. JAMA 284: 835-842.

3. Puymirat, Etienne, Marc Bonaca, Maxime Fumery, Victoria Tea, et al. (2019) Atherothrombotic Risk Stratification after Acute Myocardial Infarction: The Thrombolysis in Myocardial Infarction Risk Score for Secondary Prevention in the Light of the French Registry of Acute ST Elevation or Non-ST Elevation Myocardial Infarction Registries. Clin Cardiol 42: 227-234.

4. Eagle KA, Lim MJ, Dabbous OH, Pieper KS, Goldberg RJ, et al. (2009) A validated prediction model for all forms of acute coronary syndrome: estimating the risk of 6-month postdischarge death in an international registry. JAMA 291: 2727-2733.

5. Pennell DJ (2003) Cardiovascular Magnetic Resonance: Twenty-First Century Solutions in Cardiology. Clinical Medicine. 3: 273-278.

6. Serrao GW, Lansky AJ, Mehran R, Stone GW (2018) Predictors of Left Ventricular Ejection Fraction Improvement After Primary Stenting in ST-Segment Elevation Myocardial Infarction (from the Harmonizing Outcomes with Revascularization and Stents in Acute Myocardial Infarction Trial). Am J Cardiol 121: 678-683.

7. Hendriks T, Hartman M, Vlaar P, Prakken N, van der Ende Y, et al. (2017) Predictors of left ventricular remodeling after ST-elevation myocardial infarction. Int J Cardiovasc Imaging 33: 1415-1423.

8. Chia S, Senatore F, Raffel OC, Lee H, Wackers FJ, et al. (2008) Utility of cardiac biomarkers in predicting infarct size, left ventricular function, and clinical outcome after primary percutaneous coronary intervention for ST-segment elevation myocardial infarction. JACC 1: 415-423.

9. Rodrigo R, Hasson D, Prieto JC, Dussaillant G, Ramos C, et al. (2014) The effectiveness of antioxidant vitamins $\mathrm{C}$ and $\mathrm{E}$ in reducing myocardial infarct size in patients subjected to percutaneous coronary angioplasty (PREVEC Trial): study protocol for a pilot randomized double-blind controlled trial. Trials 15: 192.

10. Antman, Elliott M, Daniel T Anbe, Paul Wayne Armstrong, Eric R Bates, et al. (2004) ACC/AHA Guidelines for the Management of Patients with ST-Elevation Myocardia Infarction--Executive Summary. A Report of the American College of Cardiology/ American Heart Association Task Force on Practice Guidelines (Writing Committee to Revise the 1999 Guidelines for the Management of Patients with Acute Myocardial Infarction. Circulation 44: 671-719.

11. Vick GW (2009) The gold standard for noninvasive imaging in coronary heart disease: magnetic resonance imaging. Curr Opin Cardiol 24: 567-579.

12. Pickett CA, Cheezum MK, Kassop D, Villines TC, Hulten EA (2015) Accuracy of cardiac CT, radionucleotide and invasive ventriculography, two- and three-dimensional echocardiography, and SPECT for left and right ventricular ejection fraction compared with cardiac MRI: a meta-analysis. Eur Heart J Cardiovasc Imaging 16: 848-852.

13. Pickett CA, Cheezum MK, Kassop D, Villines TC, Hulten EA (2015) Accuracy of cardiac CT, radionucleotide and invasive ventriculography, two- and three-dimensional echocardiography, and SPECT for left and right ventricular ejection fraction compared with cardiac MRI: a meta-analysis. Eur Heart $J$ Cardiovasc Imaging 16: 848-852. [Crossref]

14. Foley, Thomas A., Sunil V Mankad, Nandan S Anavekar, Crystal R Bonnichsen, et al. (2012) Measuring Left Ventricular Ejection Fraction - Techniques and Potential Pitfalls. ECR 8: 108-114 
Valle-Batalla LD (2019) Validation of myocardial damage biomarkers as reliable predictors of infarct size and ejection fraction impairment assessed by cardiac magnetic resonance

15. Apple FS, Sharkey SW, Falahati A, Murakami M, Mitha N, et al. (1998) Assessment of left ventricular function using serum cardiac troponin I measurements following myocardial infarction. Clinica chimica acta 272: 59-67.

16. Wu W, Li DX, Wang Q, Xu Y, Cui YJ (2018) Relationship between high-sensitivity cardiac troponin $\mathrm{T}$ and the prognosis of elderly inpatients with non-acute coronary syndromes. Clinical Interventions in Aging 13: 1091-1098.

17. Reinstadler SJ, Feistritzer HJ, Klug G, Mair J, Tu AMD, et al. (2016) High-sensitivity troponin $\mathrm{T}$ for prediction of left ventricular function and infarct size one year following ST-elevation myocardial infarction. International Journal of Cardiology 202: 188-193.
18. Eggers KM, Lindahl B (2017) Prognostic Biomarkers in Acute Coronary Syndromes Risk Stratification Beyond Cardiac Troponins. Current Cardiology Reports 19: 29.

19. Sawaya H, Sebag IA, Plana JC, Januzzi JL, Ky B, et al. (2012) Assessment of echocardiography and biomarkers for the extended prediction of cardiotoxicity in patients treated with anthracyclines, taxanes, and trastuzumab. Circ Cardiovasc Imaging 5: 596-603.

20. Cediel G, Rueda F, García C, Oliveras T, Labata C, Serra J, et al. (2017) Prognostic Value of New-Generation Troponins in ST-Segment-Elevation Myocardial Infarction in the Modern Era: The RUTI-STEMI Study. JAHA 6: 1-10.

Copyright: (C2019 Valle-Batalla LD. This is an open-access article distributed under the terms of the Creative Commons Attribution License, which permits unrestricted use, distribution, and reproduction in any medium, provided the original author and source are credited. 